\title{
Atuação multiprofissional à mulher vítima de violência doméstica: assistência da Saúde da Família
}

\author{
Multiprofessional care for women victims of domestic violence: family health care
}

\author{
Atención multiprofesional a mujeres víctimas de violencia doméstica: atención de salud \\ familiar
}

Caroline Silva de Araujo Lima1*, Ana Caroline Grilo de Paiva², Isadora Santos Aleixo³, Lucas Alves Bandeira ${ }^{4}$, Manoella Gotardo Aguiar Gurgel ${ }^{5}$, Maria Eduarda Fraga Nogueira ${ }^{6}$, Poliana de Faria Miziara Jreige ${ }^{4}$, Sebastião Barreto Falcão Neto ${ }^{7}$, Vilma Raquel Lima Ramalho de Holanda ${ }^{8}$, Mônica Isaura Corrêa9 .

\section{RESUMO}

Objetivo: Identificar o papel da equipe de saúde da família no acolhimento às vítimas de violência doméstica e descrever os empecilhos presentes no serviço disponível. Revisão bibliográfica: É de suma importância que os profissionais da saúde tenham uma escuta qualificada e atenciosa, ofertando às vítimas uma garantia de confidencialidade possibilitando o relato da violência vivida. Além disso, reforça-se a importância do cuidado multidisciplinar, de forma a atender as demandas da vítima de maneira íntegra. Porém, a falta de articulação entre os serviços e entre os profissionais, a inadequação de estrutura física e a dificuldade de busca de uma rede de apoio são dificuldades encontradas pelas vítimas de violência doméstica, assim como, a falta de um protocolo e a revitimização. O despreparo acerca da temática devido a lacunas na formação do profissional de saúde e processos de qualificação incipiente, acarretam consequências negativas, como abordagem inadequada e práticas preconceituosas, fragilizando ainda mais a mulher. Considerações finais: Faz-se necessário que as UBS promovam ações de saúde, a capacitação dos profissionais da APS e a prevenção, ofertando serviços que auxiliem e acolham mulheres em situações de violência.

Palavras-chave: Saúde da mulher, Violência doméstica, Equipe de assistência ao paciente.

\begin{abstract}
Objective: To identify the role of the family care team in welcoming victims of domestic violence and describe the obstacles present in the available service. Bibliographic review: It is of utmost importance that health professionals have a qualified and attentive listening, offering victims a safe place so they can entrust the story about the violence that they experienced. In addition, it is necessary that all the demands mentioned by the woman are fully attended to, thus portraying the protection and care by qualified professionals, and for this a multidisciplinary team is necessary. However, the lack of coordination between services, professionals, the inadequacy of physical structure and the difficulty to finding a support network are obstacles encountered by victims of domestic violence, as well as the lack of a protocol and the revictimization. The unpreparedness on the subject due to gaps in the training of health professionals and incipient qualification processes, has negative consequences, such as inadequate approach and prejudiced practices, further weakening the victims. Final considerations: It is necessary that the Basic Health Unit promote health actions, the training of professionals and prevention, offering services that help and welcome women in situations of violence.
\end{abstract}

Keywords: Women's health, Domestic violence, Patient care team.

\footnotetext{
${ }^{1}$ Faculdade Dinâmica Vale do Piranga (FADIP), Ponte Nova - MG. *E-mail: carolinearaujo689@gmail.com

2 Universidade Nove de Julho (UNINOVE), São Paulo - SP.

${ }^{3}$ Centro Universitário de Belo Horizonte (UniBH), Belo Horizonte - MG.

${ }^{4}$ Centro Universitário do Planalto Central Apparecido dos Santos (UNICEPLAC), Brasília - DF.

5 Universidade de Fortaleza (UNIFOR), Fortaleza - CE.

${ }^{6}$ Centro Universitário do Espírito Santo (UNESC), Colatina - ES.

7 Universidade Federal do Amazonas (UFAM), Manaus - AM.

${ }^{8}$ Centro Universitário de Patos (UNIFIP), Patos - PB.

${ }^{9}$ Faculdade de Medicina do Vale do Aço (UNIVAÇO), Ipatinga - MG.
} 


\section{RESUMEN}

Objetivo: Identificar el papel del equipo de salud familiar en la acogida de las víctimas de la violencia doméstica y describir los obstáculos presentes en el servicio disponible. Revisión bibliográfica: Es de suma importancia que los profesionales sanitarios dispongan de un servicio de escucha cualificado y atento, que ofrezca a las víctimas una garantía de confidencialidad que permita denunciar la violencia vivida. Además, es necesario que todas las causas referidas por la mujer sean atendidas de manera integral, retratando así, la protección y atención por parte de profesionales calificados, y para ello es necesario un equipo multidisciplinario. Sin embargo, la falta de coordinación entre los servicios y los profesionales, la estructura física inadecuada y la dificultad para encontrar una red de apoyo son dificultades que encuentran las víctimas de la violencia doméstica, así como la falta de un protocolo y de revitalización. La falta de preparación sobre el tema debido a las lagunas en la formación del profesional de la salud y los procesos de calificación incipiente, traen consigo consecuencias negativas, como un enfoque inadecuado y prácticas prejuiciosas, lo que hace que las mujeres sean aún más frágiles. Consideraciones finales: Por lo tanto, es necesario que la UBS promueva acciones de salud, formación de profesionales de la APS y prevención, ofreciendo servicios de ayuda y acogida a las mujeres en situaciones de violencia.

Palabras clave: Salud de la mujer, Violencia doméstica, Grupo de atención al paciente.

\section{INTRODUÇÃO}

A violência doméstica contra mulher (VDCM) é considerada uma violação dos direitos humanos que atinge mulheres por todo o mundo. Diz respeito a todo ato de violência contra mulher, que lhes cause sofrimento psicológico, físico, sexual, financeiro ou emocional, impactando-as no que tange aos direitos à vida, à saúde e à integridade física e mental (ARBOIT J, et al., 2018).

A violência é um fenômeno que atinge mulheres de diferentes orientações sexuais, classes sociais, origens, regiões, estados civis, escolaridade ou raças/etnias em relações desiguais de poder. Pode ocorrer desde a infância até a velhice, seja no campo do trabalho, nas dimensões religiosas, culturais e/ou comunitárias, entre outras (BRASIL, 2016).

A VDCM é um fenômeno sociocultural, decorrente de uma sociedade baseada nas relações de poder e conflito entre os gêneros. Dá-se pela interação de fatores individuais, relacionais, sociais, culturais e ambientais, que estão ligadas às questões de gênero de uma ordem social de tradição patriarcal, uma vez que a violência ocorre devido à posição desigual das mulheres nos relacionamentos e a "legitimidade" que o homem tem em controlar os bens e os comportamentos dessa mulher. Assim, a violência se estabelece quando a mulher desafia este controle ou o homem não consegue mantê-lo (SANTOS WJ, et al., 2018; LEITE FMC, et al., 2017).

A categorização da VDCM como problema de saúde pública não se faz necessária somente pela magnitude das estatísticas envolvidas, mas também pela identificação dos efeitos das relações e situações de violência como um grande impeditivo para o desenvolvimento humano (FERNANDES SILVA AC, et al., 2020).

Entre as consequências da VDCM para saúde das vítimas, observa-se o intenso prejuízo à saúde mental destas. Os problemas psiquiátricos que vão desde transtornos mentais comuns como insônia, fadiga, irritabilidade, esquecimento, dificuldade de concentração e queixas somáticas até transtornos depressivos, ansiedade, transtorno de estresse pós-traumático, transtorno somatoforme e de personalidade (ARBOIT J, et al., 2017).

Aliado a esses transtornos, há o aumento do uso de substâncias lícitas e ilícitas, bem como de medicamentos psicotrópicos e analgésicos. Evidencia-se ainda que estes transtornos são uma causa importante de dias perdidos de trabalho e, consequentemente, representam um alto custo social e econômico (ARBOIT J, et al., 2017).

Dados de 2013 da Organização Mundial da Saúde, apontam que 30\% das mulheres no mundo já foram vítimas de violência doméstica. No Brasil, de acordo com dados das Delegacias Especializadas no Atendimento a Mulheres que constam no Mapa da Violência 2015, são notificadas cerca de 205 mil agressões 
a mulheres no período de um ano. Além disso, outro estudo de base populacional realizado num período de 15 anos, evidenciou que $43 \%$ das mulheres brasileiras afirmaram já ter sofrido violência cometida por um homem na vida (SANTOS IB, et al., 2020).

Devido às graves consequências que podem acarretar na vida da mulher, esse fenômeno reconhecido como um problema de saúde pública tem despertado o interesse da sociedade. Uma vez que além do impacto direto sobre a saúde da mulher, essa alta prevalência de violência pode chegar a causar morte decorrente das lesões e traumas físicos e emocionais. (LUCENA KDT, et al., 2017).

Diante do exposto, torna-se evidente a importância da adequada assistência às vítimas de VDCM, uma vez que tais agressões acarretam em grandes impactos em suas vidas. Sendo assim, o presente trabalho tem como objetivo identificar o papel da equipe de saúde da família no acolhimento a essas vítimas de violência doméstica, bem como descrever os empecilhos presentes nos serviços disponíveis.

\section{REVISÃO BIBLIOGRÁFICA}

\section{Condutas desenvolvidas pela atenção primária a mulheres violentadas}

No Brasil, as Unidades Básicas de Saúde (UBS) são a porta de entrada da população à Atenção Primária, interligado a ações de promoção, prevenção e tratamento de doenças e fatores psicossociais. $A$ atenção às mulheres vítimas de violência acontece mediante a interação de uma rede organizada, com oferta qualificada de serviços que acolhem e auxiliam àquelas que buscam ajuda e necessitam de um atendimento qualificado e especializado nos cuidados integrais (OPAS, et al., 2010).

O medo, implantado pela sociedade patriarcal em busca do poder sobre as mulheres, é uma das causas pela qual a violência doméstica é encoberta. Em virtude desse medo, bem como de ameaças que as calam, dificulta-se o acesso e acolhimento nas UBS. Um estudo realizado com profissionais da Atenção Primária da Catalunha e Costa Rica demonstrou a importância de profissionais da saúde realizarem uma escuta qualificada e atenciosa, oferecendo a essas mulheres uma garantia de confidencialidade e possibilitando 0 relato sobre a violência vivida (LORÍA KR, et al., 2015).

Por se tratar de um trauma, a violência doméstica ocasiona diversas consequências, tanto físicas como psicológicas. Com isso, as UBS devem ser orientadas com enfoque na atenção intersetorial e multiprofissional, com profissionais qualificados, de forma a oferecer um serviço amplo e unido para atender essa vítima em todas as suas demandas de maneira integral (GARCÍA-MORENO C, et al.,2015; VIEIRA LB, et al., 2016).

Uma das maneiras de se coibir a violência é tirá-la do silêncio. Entre as mais importantes políticas públicas destacadas pelo movimento feminista brasileiro, está a criação das Delegacias Especiais das Mulheres. Dialogando com as delegacias especiais dentro da rede de proteção às mulheres em situação de violência, figuram as unidades de saúde e a Atenção Básica. Elas ocupam papel importante na luta contra todo e qualquer tipo de violência, que podem influenciar ou causar processos de adoecimento, derivando em questões de saúde pública (BRASIL, 2016).

Por se tratar de um problema que assola a sociedade, a violência contra a mulher requer a interlocução de vários setores de assistência, visando à desconstrução de convenções implantadas sobre a figura feminina. Para isso, é imprescindível investimentos destinados a ações conjuntas de saúde pública, a fim de oferecer um suporte qualificado e profissionais aptos a atender e orientar a vítima. Diante disso, faz-se necessário um arranjo intersetorial no intuito de buscar por aperfeiçoamentos tanto de profissionais da atenção primária como no ambiente de acolhimento, para que o serviço de saúde seja capaz de atender de forma equitativa e integral à população, denotando a importância do papel das UBS, favorecendo assim, a obtenção do relato desconstruindo o silêncio enfrentado pelas vítimas (HERLER LZ, et al., 2013; BAIRMERRITT MH, et al., 2014; OLIVEIRA RNG, 2014).

O Ministério da Saúde publicou em 2001 a Portaria no 737, que institui a Política Nacional de Redução da Morbimortalidade por Acidentes e Violências. Com isso, o enfrentamento da violência segue, algumas 
diretrizes, como a promoção da adoção de comportamentos e de ambientes seguros e saudáveis; monitorização da ocorrência de acidentes e de violências; assistência interdisciplinar e intersetorial às vítimas de acidentes e violências; entre outras (HERLER LZ, et al., 2013; BAIR-MERRITT MH, et al., 2014; OLIVEIRA RNG, 2014).

Logo, em 2006 a Política Nacional de Promoção da Saúde (PNSP) o qual foi instituída pela Portaria no 687, foi revisada em 2014. A PNPS tem, como um de seus eixos, a cultura da paz e dos direitos humanos. Dessa forma , com o pacto destacam-se duas leis: i) a Lei ํㅜ 10.778/2003,6 que estabelece a notificação compulsória em território nacional dos casos de violência contra a mulher que for atendida em serviços de saúde, públicos ou privados; e ii) a Lei no 11.340/2006, conhecida como Lei Maria da Penha, que define a violência "doméstica e familiar contra a mulher" como qualquer ação ou omissão baseada no gênero que lhe cause morte, lesão, sofrimento físico, sexual ou psicológico e dano moral ou patrimonial sendo responsável por medidas penais para a contenção da violência (BRASIL, 2003; BRASIL, 2006).

Dessa forma, especificamente no setor saúde, as instituições envolvidas na atenção às pessoas em situação de violência sexual devem assegurar cada etapa do atendimento que for necessária. Isso inclui medidas de prevenção, emergência, acompanhamento, reabilitação, tratamento de eventuais agravos e impactos resultantes da violência sobre a saúde física e psicológica, além do abortamento legal se for solicitado pela mulher, de acordo com a legislação vigente (BRASIL, 2016).

Destacam-se como legislação para essa pauta a Lei oㅡ 12.845/2013, que dispõe sobre o atendimento obrigatório e integral de pessoas em situação de violência sexual, o Decreto $n^{\circ}$ 7.958/2013, que estabelece diretrizes para o atendimento às vítimas de violência sexual pelos profissionais de segurança pública e da rede de atendimento do SUS e a Norma Técnica Prevenção e Tratamento de Agravos Resultantes da Violência Sexual Contra as Mulheres e Adolescentes do Ministério da Saúde (BRASIL, 2016).

A notificação de violência doméstica, sexual e outras violências foi universalizada pela Portaria no 104/2011, sendo em 2014, substituída pela Portaria no 1.271 , que estabeleceu também a notificação imediata dos casos de violência sexual em âmbito municipal (BRASIL, 2016).

\section{O papel da estratégia de saúde da família}

Segundo a Política Nacional de Atenção Básica (PNAB), a atenção básica tem como princípios fundamentais a formulação de ações, individuais ou coletivas, para a promoção de saúde, prevenção de agravos, diagnóstico, tratamento e reabilitação. No entanto, no Brasil, a VDCM possui um contexto de difícil combate formado por bases históricas de uma sociedade fundamentada em relações de poder patriarcais. Esse contexto pode acabar afastando da conflituosa relação familiar a possível influência dos agentes promotores de saúde, constituindo, dessa forma, um desafio para as instituições de saúde, sobretudo em nível de atenção básica, diagnosticar esse agravo e combatê-lo de maneira categórica (SANTOS IB, et al.,2020).

Dessa forma, torna-se fundamental que a atenção à saúde dessas mulheres aconteça no âmbito da Rede de Atenção à Saúde. Entende-se que no setor da saúde, principalmente no contexto da Atenção Primária à Saúde (APS), os profissionais se encontrem em uma posição estratégica para assistir às mulheres em situação de violência, uma vez que a APS é considerada como reorientadora do sistema, e não apenas um nível dele (SANTOS IB, et al.,2020).

É importante ressaltar que os profissionais, não somente o médico, têm como objetivo constituir o centro de comunicação, ordenar, organizar fluxos, contrafluxos de usuários, produtos e informações, ao longo de todos os pontos que a formam, de modo a promover uma atenção qualificada, integral e que contemple as necessidades das usuárias, especialmente, aquelas direcionadas às mulheres em situação de violência, denotando a importância do acolhimento, escuta e acompanhamento (ARBOIT J, et al., 2017).

Pelo fato de a Atenção Básica estar presente em todos os setores de saúde, torna-se mais próxima do cotidiano das pessoas, o qual tem papel importante na identificação de situações de violência e, assim, o contato com as primeiras abordagens realizadas com as mulheres, além da promoção do cuidado e do acesso a informações sobre serviços da rede que possam apoiá-las (BRASIL, 2016). 
Assim, é imprescindível compreender que fatores biopsicossociais também contribuem para o contexto de difícil combate da VDCM no Brasil. Pode-se afirmar que há, nesse contexto, uma "rotinização da violência", em que as promessas do companheiro de não mais agredir, a falta de condições para uma mudança de vida da vítima, a vergonha e a falta de apoio da própria família constituem subsídios para o aumento da tolerância das vítimas a esses agravos. Destaca-se, portanto, a importância da descentralização e capilarização das ações das equipes de saúde com o intuito de diagnosticar e romper o ciclo de rotinização da violência objetivando a notificação desse agravo, observando o cuidado integral à saúde da vítima tanto no âmbito físico quanto psicológico (SILVA ACF, et al., 2020).

Nesse sentido, torna-se de fundamental importância a observação das atribuições de enfermeiros, auxiliares e técnicos de enfermagem, médicos, agentes comunitários de saúde, cirurgiões-dentistas e técnicos e auxiliares em saúde bucal expressas na PNAB. Assim, a partir da compreensão do contexto de difícil combate a VDCM no Brasil, esses profissionais de saúde devem promover um acolhimento com escuta qualificada, atentando-se para a presença de sinais e sintomas clínicos de agressão física e psicológica, situações de risco e vulnerabilidade, além de coletar informações e promover a notificação do agravo (SILVA ACF, et al., 2020).

Para isso, a equipe de atenção básica de saúde deve realizar um trabalho interdisciplinar e em equipe objetivando somar a expertise de cada profissional que a compõe e, utilizando-se das informações obtidas a partir da escuta qualificada, realizar o encaminhamento intersetorial de forma adequada para unidades como os Centros de Referência de Atenção à Saúde da Mulher em situação de violência (CRAM) (BRASIL, 2012).

Destaca-se a importância do papel do agente comunitário de saúde (ACS) no contexto do combate a VDCM, visto que a elevada capilaridade inerente a suas atribuições aumenta a possibilidade de identificação da VDCM, sobretudo a partir das visitas domiciliares. A partir das visitas, o ACS pode identificar fatores que, devido a "rotinização da violência", são de difícil observação, como a relação familiar em domicílio, relatos de violência doméstica a partir de familiares e vizinhos, sinais de agressão e demais fatores que podem contribuir para a violência doméstica ou para a dificuldade em diagnosticá-la (SILVA ACF, et al., 2020).

É importante que ao realizar a visita domiciliar, os profissionais de saúde partam do princípio que a VDCM tende a ser um agravo com elevada subnotificação e, portanto, é indispensável realizar ações de prevenção, educação e tratamento, tanto nas unidades básicas de saúde como nos domicílios e pontos de apoio do território adscrito (SILVA ACF, et al., 2020).

Ademais, cabe aos médicos e aos enfermeiros da Estratégia de Saúde da Família exercerem práticas de acolhimento a partir da escuta qualificada de maneira a manter o vínculo de confiança construído a partir das visitas domiciliares, ações educativas ou diálogo promovido por outros membros das equipes de saúde objetivando a resolutividade do agravo de forma integral, observando aspectos físicos, psicológicos e sociais do usuário. Para isso, é necessário reconhecer os limites de cada usuário em relação a VDCM, compreendendo que o medo e o abalo psicológico são características presentes durante o processo de acolhimento (MARTINS LCA, et al., 2016).

Nesse sentido, esses profissionais de saúde devem estar capacitados para repassar informações relevantes, de maneira particular e individualizada, e colher dados como sinais físicos de agressão, tais como hematomas, lesões, escoriações e fraturas bem como sinais psicológicos de agressão, tais como tremores, ansiedade, insônia e perda de peso. A partir do vínculo criado entre usuário e equipe de saúde e da coleta de informações, é possível criar ações de resolutividade tanto no âmbito individual quanto no âmbito coletivo, uma vez que, a partir das informações obtidas, é possível identificar áreas de maior vulnerabilidade a esse agravo dentro do território adscrito pela unidade de saúde (MARTINS LCA, et al., 2016).

Com isso, as equipes podem formular ações educativas e preventivas com a ajuda das redes de apoio existentes com o intuito de arrefecer os índices de VDCM e fortalecer o vínculo entre os profissionais das Equipes de saúde da Família e os usuários do sistema de saúde (MARTINS LCA, et al., 2016).

Em relação à visita domiciliar, estudos evidenciam o benefício desta atividade a curto prazo, demonstrando eficácia na diminuição da violência por parceiro íntimo e no acompanhamento das mulheres que a vivenciam. Dessa forma, os Agentes comunitários de saúde possuem um importante papel na criação de estratégias que 
visam reduzir os episódios de violência doméstica contra mulheres em suas áreas de atuação. E, para que isso se torne possível, torna-se necessário o apoio de outros membros da equipe, como por exemplo, o enfermeiro, que podem capacitá-las para realizar a identificação e o encaminhamento das vítimas de violência (ARBOIT J, et al., 2017).

\section{Empecilhos presentes nos serviços de saúde}

A assistência às vítimas de violência doméstica exige qualidade da atenção e articulação em rede de diversos serviços intersetoriais prestados às mulheres que vivenciam a violência, a saber: Secretaria de Saúde, Secretaria de Assistência Social, UBS, Centros de Atenção Psicossocial, delegacia da mulher, Centro Especializado de Assistência Social (CREAS), Pronto Atendimento e casa de passagem. É possível observar que a assistência de equipe multidisciplinar traz consigo potencialidades na busca da criação de vínculos e assistência adequada a estas mulheres (TRENTIN D, et al., 2020).

Entretanto, ocorre uma falta de articulação entre os serviços, devido tanto ao desconhecimento e ausência de profissionais, bem como pela falta de comunicação entre as partes, inadequação da estrutura física e dificuldade de busca de uma rede de apoio (TRENTIN D, et al.,2020). Para Arboit J, et al. (2017) a desarticulação de serviços é empecilho para a assistência da mulher em situação de violência. Essa desorganização leva os trabalhadores da APS a não identificarem com quais serviços podem contar para desenvolver ações de atenção a essa população.

Mesmo com elevada prevalência e risco de violência, a atenção em saúde ofertada às mulheres em situação de violência de gênero, ainda é insatisfatória. Essa situação decorre da invisibilidade do fenômeno em alguns setores, como os hospitais de urgência, que em sua maioria ainda não possuem instrumentos capazes de identificar o problema, evidenciando a predominância do modelo biologicista de assistência à saúde, cujo objeto de intervenção é o dano físico (LUCENA KDT, et al., 2017).

Outra dificuldade presente nos serviços é a revitimização. De acordo com Jaqueline A, et al. (2017) para que o acolhimento se torne efetivo é necessária a atuação de equipe multidisciplinar, cuja função é acolher a vítima e garantir acesso aos serviços da rede de apoio. Desse modo, segundo orienta o Protocolos da Atenção Básica: saúde das mulheres (2016), a coleta de relato do caso só deverá ser realizada se o profissional tiver conhecimento das condutas de acolhimento necessárias à saúde da mulher e da articulação intersetorial adequada à condução do caso. Com o fito de evitar a narração e a exposição da violência repetidas vezes .

Sem esse manejo oportuno, realizar o encaminhamento a um profissional adequado para cada tipo de problema pode aparentar ser um descaso com a vítima, visto que ao chegar a outro profissional o processo se reiniciará com a contagem de sua história e todos os procedimentos de acolhimento e confiança. Nesse sentido, o encaminhamento dessas mulheres a serviços que não dialogam entre si as expõem a um processo traumático de fragmentação do cuidado e de revitimização, em que a usuária precisa relatar novamente sua vivência a cada serviço ou profissional que procura (JAQUELINE A, et al., 2017; TRENTIN D, et al., 2020).

Sabe-se que apesar da existência da Lei Maria da Penha, muitas mulheres não se sentem seguras e protegidas para denunciar o agressor. Essa insegurança se relaciona à dificuldade de reagir e verbalizar a violência, o temor pela própria segurança e dos filhos, a falta de controle sobre suas vidas, a esperança de que agressor mude ou a disposição para proteger o parceiro seja por motivos econômicos ou afetivos. (LUCENA KDT, et al., 2017).

Para que o encaminhamento se torne um instrumento de potencializar as ações prestadas pelos profissionais desta rede, é necessária uma maior articulação entre os serviços. Tais serviços não envolvem apenas o âmbito da área de saúde, mas se estendem a outros profissionais como psicólogos e assistentes sociais, ajudando a diminuir os casos de revitimização, que é um dos cenários ainda presentes na vida de várias mulheres (JAQUELINE A, et al., 2017; TRENTIN D, et al., 2020).

É importante destacar que os entraves de cunho institucional se referem à ineficiência do sistema em garantir a proteção da mulher diante de seu agressor, o despreparo e a burocracia do sistema jurídico e legal, o despreparo de profissionais de saúde para lidar com situações de violência e a falta de acompanhamento e de responsabilização (LUCENA KDT, et al., 2017). 
Ademais, o despreparo acerca da temática devido a lacunas na formação do profissional de saúde e processos de qualificação incipiente acarretam consequências negativas como abordagem inadequada e práticas preconceituosas, fragilizando ainda mais a mulher (ARBOIT J, et al.,2018; TRENTIN D, et al., 2020).

\section{CONSIDERAÇÕES FINAIS}

Diante dos fatos supracitados, este estudo evidenciou a violência doméstica como um importante problema de saúde pública, uma vez que resulta em grandes impactos na saúde física e psicológica das vítimas. Além disso, apontou alguns empecilhos na identificação e na conduta das dessas mulheres, como a ausência de um protocolo, a falta de articulação entre os serviços e a inadequação de uma estrutura física para acolhimento. Desse modo, a fim de atender ao princípio de integralização do Sistema Único de Saúde (SUS), as UBS devem promover ações de saúde, a capacitação dos profissionais da APS e a prevenção, ofertando serviços que auxiliem e acolham mulheres em situações de violência.

\section{REFERÊNCIAS}

1. ARBOIT J, et al. Atenção à saúde de mulheres em situação de violência: desarticulação dos profissionais em rede. Revista da Escola de Enfermagem da USP, 2017; 51.

2. ARBOIT J, et al. Violence against women in primary health care: Potentialities and limitations to identification. Atencion Primaria, 2020; 52(1): 14-21.

3. BARROS CRS, SCHRAIBER LB. Violência por parceiro íntimo no relato de mulheres e de homens usuários de unidades básicas. Revista de Saúde Pública, 2017; 51(7).

4. BAIR-MERRITT MH, et al. Primary care-based interventions for intimate partner violence: a systematic review. Am J Prev Med. 2014; 46(2): 188-94.

5. BRASIL. Manual do Ministério da Saúde. 2012. Disponível em: http://189.28.128.100/dab/docs/publicacoes/geral/pnab.pdf. Acessado em: 27 de abril de 2021.

6. BRASIL. Manual do Ministério da Saúde. 2016 Instituto Sírio-Libanês de Ensino e Pesquisa. Protocolos da Atenção Básica: saúde das mulheres. Brasília (DF): Ministério da Saúde.

7. BRASIL. Ministério da Saúde. Gabinete do Ministro. Portaria № 737, de 16 de Maio de 2001. Brasília, 2001. Disponível em: http://bvsms.saude.gov.br/bvs/saudelegis/gm/2001/prt0737_16_05_2001.html

8. BRASIL. Lei no 10.778, de 24 de novembro de 2003. Estabelece a notificação compulsória, no território nacional, do caso de violência contra a mulher que for atendida em serviços de saúde públicos ou privados. Brasília, 2003. Disponível em: <http://www.planalto.gov.br/

9. ccivil_03/leis/2003//10.778.htm>.

10. BRASIL. Lei no 11.340, de 7 de agosto de 2006 (Lei Maria da Penha).

11. GARCÍA-MORENO C Hegarty K, et al. The health-systems response to violence against women. Lancet. 2015; 385(9977): 1567-79.

12. HESLER LZ, et al. Violence against women in the perspective of community health agents. Rev Gaúcha Enferm, 2013; 34(1): 180-6.

13. LEITE MFC, et al. Violência contra a mulher em Vitória, Espírito Santo, Brasil. Revista de Saúde Pública, $2017 ; 51: 33$.

14. LUCENA KDT, et al. Associação entre a violência doméstica e a qualidade de vida das mulheres. Revista Latino-Am. Enfermagem, 2017; 25.

15. LORÍA KR, et al. Actitud hacia la violencia de género de los profesionales de Atención Primaria: estudio comparativo entre Catalunã y Costa Rica. Atenção Prim. 2015; 47(8): 490-7.

16. MARTINS LCA, et al. Violência contra mulher: acolhimento na estratégia saúde da família. Ciência, Cuidado e Saúde, 2016; 15(3): 507-514.

17. MENDONÇA MFS, LUDEMIR AB. Violência por parceiro íntimo e incidência de transtorno mental comum. Revista de Saúde Pública, 2017; 51:32.

18. ORGANIZACIÓN PANAMERICANA DE SALUD (OPAS). Redes Integradas de Servicios de Salud. Conceptos, opciones de política y hoja de ruta para suimplementación en las Américas [Internet]. Washington: OPAS; 2010.

19. OLIVEIRA RNG, FONSECA RMGS. A violência como objeto de pesquisa e intervenção no campo da saúde: uma análise a partir da produção do grupo de pesquisa gênero, saúde e enfermagem. Rev Escola Enferm USP [online]. 2014 dez [citado 2016 out $181 ; 48$ (Esp 2):32-9.

20. OLIVE P. First contact: acute stress reactions and experiences of emergency department consultations following an incident of intimate partner violence. Journal of Clinical Nursing, 2016;

21. SANTOS IB, et al. Violência contra a mulher a mulher na vida: estudo entre usuárias da Atenção Primária. Ciência e Saúde Coletiva, 2020; 25(5): 1935-1946.

22. SANTOS WJ, et al. Violência doméstica contra a mulher perpetrada por parceiro íntimo: representações sociais de profissionais da atenção primária à saúde. Rev Fund Care Online, 2018; 10(3): 770-777.

23. SILVA ACF, et al. Violência sexual por parceiro íntimo identificada em Unidade Básica do PSF. Revista Nursing, $2020 ; 23$ (263): 3705-3709.

24. TRENTIN D, et al. Mulheres em situação de violência sexual: potencialidades e fragilidades da rede intersetorial. Revista Brasileira de Enfermagem, 2020; 73(4).

25. VIEIRA LB, Souza IE de O, Tocantins FR, Pina-Roche F. Support to women who denounce experiences of violence based on her social network. Rev Latino Am Enfermagem [Internet]. 2015 [cited 2016 June 13];23(5):865-73.

26. WAISELFISZ JJ. Mapa da Violência 2015: Homicídio de Mulheres no Brasil. 1. ed. Brasília (DF): 2015.

27. WILLIAMS JR, et al. An exploration of screening protocols for intimate partner violence in healthcare facilities: a qualitative study. Journal of Clinical Nursing, 2016; 26: 2192-2201. 